\title{
Performance de agências bancárias: aplicando DEA a múltiplas perspectivas do desempenho
}

Performance of banks agencies: applying DEA to multiples performance perspective

\section{Marcelo Alvaro da Silva Macedo}

Doutor em Engenharia de Produção na COPPE pela Universidade Federal do Rio de Janeiro

Professor do Mestrado em Ciências Contábeis da FACC - Universidade Federal do Rio de Janeiro

Endereço: Depto. de Contabilidade - FACC - UFRJ

Av. Pasteur, 250 - Urca

CEP: 22.290-902 - Rio de Janeiro/RJ

E-mail: malvaro.facc.ufrj@gmail.com

Telefone: (21) 9765-6553

\section{Glaydson Teixeira Cavalcante}

Mestre em Gestão e Estratégia em Negócios pela Universidade Federal Rural do Rio de Janeiro

Endereço: Rod. BR 465, Km 07 - PPGEN/UFRRJ

CEP: 23.890-000 - Seropédica/RJ

Email: glaydson@bol.com.br

Telefone: (21) 26821042

Artigo recebido em julho de 2009. Passou por uma avaliação double blind review em novembro de 2009. Aceito em dezembro de 2009 pela Editora Científica Sandra Rolim Ensslin. 


\title{
$\overline{\text { Resumo }}$
}

Neste artigo, será aplicada a metodologia $D E A$ a indicadores de perspectivas balanceadas (pesos iguais) do Balanced Scorecard (BSC), com o objetivo de definir unidades eficientes e ineficientes. Para tanto, analisase 38 agências do segmento de varejo, de uma mesma instituição nacional, que é classificada como um dos maiores bancos brasileiros. Os resultados mostram o Índice de Desempenho Multicriterial - IDM, que representa o índice consolidado de desempenho multicriterial de cada um das agências sob análise, levando em consideração as perspectivas de desempenho financeira, operacional, de clientes e de pessoal. Percebe-se, ainda, ao nível de $10 \%$ de significância, que o desempenho tem relação com o tamanho das agências, pois o desempenho multicriterial médio das 10 maiores agências é superior ao das 10 menores agências. Isso é reflexo do ganho de escala que existe nas operações das agências.

Palavras-chave: Desempenho, Análise Envoltória de Dados, Balanced Scorecard.

\begin{abstract}
In this article, will be applied the DEA methodology in the balanced perspective indicators (equal weights) of the Balanced Scorecard (BSC), in order to define efficient and inefficient units. For this, we analyze 38 retail segment agencies, of a single national institution, which is ranked as one of the largest Brazilian banks. The results show the Multicriterial Performance Index - IDM, which represents the consolidated index of multicriterial performance of each agencies under analysis, considering the financial, the operational, the customer and the staff performance perspectives. We can see, yet, at the $10 \%$ level of significance, that the performance is related to the size of agencies, because the average multicriterial performance of 10 major agencies is higher than the 10 smaller agencies. This reflects the scale economies that exist in the operations of agencies.
\end{abstract}

Key words: Performance, Data Envelopment Analysis, Balanced Scorecard.

\section{Introdução}

Marr e Schiuma (2003) destacam que a medição de desempenho organizacional está no foco dos gestores de negócios e, de forma parecida, dos acadêmicos e que esta tem necessariamente uma natureza multicriterial e multidisciplinar. Já segundo Almeida et al (2004), metodologias de avaliação de desempenho sempre estiveram presentes no processo evolutivo da humanidade, sendo que estas se adaptam a finalidade e objetivos dos avaliadores.

Neely et al (2005), declaram que um sistema de mensuração de performance pode ser definido como o jogo de métricas usado para quantificar tanto a eficiência quanto a eficácia das ações das empresas. Neste sentido, Rocha et al (2005) esclarecem que os indicadores de eficiência indicarão quão bem os recursos vêm sendo usados para gerar receitas e se tem havido desperdícios de recursos. Já os de eficácia indicam o nível de atingimento dos objetivos organizacionais.

Ainda segundo Rocha et al (2005) várias perspectivas deverão ser consideradas para avaliar se o sucesso foi alcançado ou não. Portanto, continuam, é 
importante o uso de medidas objetivas, subjetivas e relativas (benchmarking), para auxiliar na decisão se determinado objetivo foi alcançado. Apesar do necessário uso de tipos variados de medidas para medir o desempenho de uma organização, seria recomendável utilizar algum tipo de metodologia que consolidasse os resultados das diversas perspectivas.

Neste sentido, o problema de pesquisa, para este trabalho, se concentra em como consolidar as várias perspectivas do desempenho organizacional para analisar a performance de agências bancárias.

Sendo assim, este estudo tem como objetivo analisar o desempenho multicriterial de agências bancárias, de um grande banco brasileiro, aplicando Análise Envoltória de Dados (DEA) às informações do primeiro semestre de 2008.

As principais questões de pesquisa são, então:

- Qual o desempenho multicriterial das agências sob análise?

- Qual a relação entre o desempenho e as perspectivas utilizadas? E com o tamanho ou nível de atividade da agência?

\section{Referencial Teórico}

\subsection{Desempenho multicriterial: o foco em múltiplas perspectivas}

Esposto et al (2002) definem que um sistema de medição de desempenho é um conjunto de processos e ferramentas para se coletar e analisar dados com o intuito de apresentar informações sobre a performance de uma unidade organizacional de interesse.

Neely et al (2005) esclarecem que a mensuração do desempenho pode ser encarada como um processo de quantificação, mas seus efeitos são para estimular a ação, através de uma atuação gerencial consistente, que leve a realização das estratégias.

Rocha et al (2005) ressaltam que o julgamento do sucesso de uma empresa depende dos critérios adotados. Isto é, a partir de alguns critérios poder-se-ia considerar uma unidade eficiente, porém a partir de critérios diferentes poderse-ia considerar esta mesma unidade como ineficiente. A definição de critérios é essencial, pois os executivos determinam suas ações futuras baseadas em como percebem e julgam o desempenho.

Isso nos remete a uma discussão interessante sobre quais indicadores utilizar e como utiliza-los no julgamento final. Neste sentido, Ferraz e Martins (2002) afirmam que o campo da mediação de performance empresarial vive uma revolução e busca de novas formas e modelos para mensuração do sucesso frente à inadequação dos modelos tradicionais, baseados apenas nas métricas financeiras. 
O método Balanced Scorecard, de 1990, desenvolvido por Kaplan e Norton, foi uma das respostas a estas preocupações, pois baseia-se no balanceamento de indicadores financeiros e não financeiros organizados em quatro perspectivas básicas: Financeira, Clientes, Processos Internos e de Aprendizado e Crescimento.

Segundo Atkinson et al. (2000), o BSC reflete a primeira tentativa sistemática de desenvolver um sistema de avaliação de desempenho que enfoque os objetivos organizacionais, a coordenação do processo decisório e a provisão de uma base para o aprendizado organizacional. Este sistema avalia os aspectos do desempenho organizacional, que sinalizam o que os gestores acreditam serem os direcionadores da performance empresarial, ou seja, as causas da eficiência ou ineficiência da empresa.

Sendo assim, ainda segundo os autores, a metodologia se apresenta como uma proposta de mensuração estruturada que complementa a abordagem tradicional de avaliação de resultado, integrando a perspectiva financeira às perspectivas representativas dos processos internos; da relação entre empresa e cliente e da capacidade da organização no que se refere ao aprendizado e crescimento.

De acordo com Kaplan e Norton (1997) a ferramenta não está restrita somente a estas quatro perspectivas, podendo a empresa, na formulação ou na reformulação de seu BSC, incluir outras perspectivas. Estes alertam aos formuladores do BSC que cuidem para não sobrecarregarem o scorecard de indicadores, o que dificultaria o acompanhamento gerencial; que escolham indicadores que sejam críticos ao sucesso e a sustentabilidade corporativa; e que estes devam mostrar as relações de causa e efeito do desempenho organizacional.

Os indicadores selecionados, continuam os autores, para cada perspectiva, devem originar-se de objetivos que respondam os seguintes questionamentos: "Para sermos bem-sucedidos financeiramente, como deveríamos ser vistos pelos nossos acionistas?" (perspectiva financeira); "Para alcançarmos nossa visão, como deveríamos ser vistos pelos nossos clientes?"(perspectiva clientes); "Para satisfazermos nossos acionistas e clientes, em que processos de negócios devemos alcançar a excelência?"(perspectiva processos internos); "Para alcançarmos nossa visão, como sustentaremos nossa capacidade de mudar e melhorar?" (perspectiva aprendizado e crescimento).

Kaplan e Norton (1997) destacam, ainda, a importância do BSC no sistema gerencial, desde que o BSC alinhe e apóie processos chaves de esclarecimento e atualização da estratégia, de comunicação da estratégia a toda a organização, de alinhamento das metas de departamentos e indivíduos à estratégia, de identificação e alinhamento das iniciativas estratégicas, de associação dos objetivos estratégicos com as metas de longo prazo e os orçamentos anuais, de alinhamento das revisões estratégicas e operacionais e de obtenção de feedback para fins de conhecimento 
e aperfeiçoamento da estratégia.

Almeida et al (2004) destacam que apesar das críticas que o BSC vem sofrendo, o Balanced Scorecard segue as tendências dos sistemas modernos de medição de desempenho, utilizando medidas balanceadas e integradas para gerenciar o alcance dos objetivos estratégicos organizacionais. Além disso, o BSC propõe uma revisão periódica da visão estratégica e a adequação das medidas de desempenho de acordo com as mudanças.

Porém, uma questão que se torna importante numa análise de desempenho apoiada em múltiplas perspectivas é como consolidar seus indicadores. Ou seja, como tomar a decisão sobre a performance de unidades sob análise tendo-se um conjunto de vetores de desempenho, sendo cada um destes representativo de um modelo monocriterial de análise do desempenho.

Para isso é preciso aplicar metodologias que integrem os indicadores de diversas naturezas, de modo a organizar e condensar a informação, haja vista a quantidade de indicadores e a diversidade de combinações possíveis para análise de desempenho. Segundo Santos e Casa Nova (2005), o que está na base de aplicação destas metodologias é o entendimento do desempenho como um fator multidimensional ou multicriterial, expresso mediante uma análise conjunta dos indicadores.

$\mathrm{O}$ uso de Métodos Multicritério de Apoio à Decisão pode ser uma solução para este tipo de problema. De acordo com Gomes et al. (2004), o Apoio Multicritério à Decisão (AMD) consiste em um conjunto de métodos e técnicas para auxiliar ou apoiar a tomada de decisões, quando da presença de uma multiplicidade de critérios.

A resposta mais importante do uso destes métodos é a caracterização de uma medida de desempenho, que faz com que a decisão fique orientada por um único indicador construído a partir de várias abordagens de desempenho diferentes. Vale ressaltar que isso facilita em muito o processo decisório, pois ao invés de considerar vários índices para concluir a respeito do desempenho da empresa o analista se utiliza apenas da medida de performance resultado do uso da metodologia multicritério.

Dentre os métodos de Apoio Multicritério à Decisão (AMD) destaca-se a Análise Envoltória de Dados (DEA), que segundo Lins e Meza (2000), apresentase como um método que fornece uma medida de desempenho capaz de comparar a eficiência de várias unidades similares mediante a consideração explícita do uso de suas múltiplas entradas para a produção de múltiplas saídas. Desta forma, esta metodologia faz com que a decisão fique orientada por um único indicador construído a partir de várias abordagens de desempenho diferentes.

Apesar de originalmente ser uma metodologia proposta em um ambiente de produção (transformação de insumos em produtos), salientam Macedo et al. 
(2006), a DEA pode ser utilizada como um método multicritério, quando se utiliza indicadores do tipo quanto menor melhor no lugar dos inputs (ex.: risco, custo, endividamento etc.) e do tipo quanto maior melhor no lugar dos outputs (ex.: lucratividade, retorno, satisfação etc.). Isso transforma a DEA em um método de Apoio Multicritério à Decisão (AMD), aplicado com o intuito de consolidar várias perspectivas (critérios) de desempenho diferentes.

A DEA já foi utilizada em vários trabalhos para consolidar medidas de desempenho organizacional, especialmente em medidas do BSC, dentre os quais destaca-se: Rickards (2003), Eilat et al. (2006), Wang (2006), Chen e Chen (2007) e Macedo et al (2008). É esta linha de pensamento que esta pesquisa procura seguir, no que diz respeito à análise do desempenho organizacional.

\subsection{Performance de agências bancárias: estudos empíricos}

A metodologia DEA, segundo Cooper et al (2004), tem sido utilizada em diversas áreas do conhecimento, devido ser uma metodologia prática para modelar processos operacionais de analise de performance. A metodologia tem sido utilizada para as mais diversas análises de desempenho em instituições financeiras, em unidades governamentais, em indústrias e comércios, no terceiro setor, etc. Lins e Meza (2000) e Macedo et al (2007) acrescentam que há interesse pela DEA dos estudiosos de diversas áreas, tais como estatística, economia, matemática, engenharia, administração, contabilidade, dentre outros.

Soteriou e Stavrinides (1997), ao revisarem o modelo de DEA para análise de serviço de qualidade em agências bancárias a partir da visão de cliente interno, concluíram que a DEA é um excelente instrumento para análise de eficiência de agências bancárias. Os autores destacam que, apesar da DEA ser um instrumento muito utilizado para análise das instituições da indústria bancária, raros são os trabalhos que utilizam esta metodologia para análise de agências bancárias.

Em seu estudo os autores analisaram o desempenho de 27 agências bancárias de porte médio de um banco europeu, utilizando como inputs horas de pessoal e de terminais de atendimento, espaço e número de contas e como outputs o nível de qualidade percebido pelos funcionários. Os resultados mostraram quais agências tinham as melhores relações entre qualidade e insumos disponíveis.

No trabalho de Avkiran (1999), tem-se a aplicação da DEA para analisar o desempenho de agências bancárias, utilizando variáveis financeiras e não financeiras. A performance de cada agência no tratamento de seus inputs (número de terminais de atendimento, número de funcionários, qualidade do atendimento) para gerar seus outputs (número de contas e resultado financeiro) foi analisada sob as perspectivas de eficiência total, técnica e de escala. Os resultados mostraram que a DEA contribui para o melhor gerenciamento de investimentos adicionais, 
racionalizando os aumentos de inputs em agências ineficientes.

Já no estudo de Camanho e Dyson (2005) fez-se a aplicação da DEA para analisar a eficiência global, técnica e de escala de agências bancárias, utilizandose como inputs informações de custos, número de terminais de atendimento, número de funcionários e como outputs total de depósitos e de empréstimos. Os resultados mostraram que a DEA foi capaz de identificar ineficiências, tais como as de escala, mostrando que algumas agências eram menores do que deveriam ser. Porém, este problema de escala não chegava afetar a lucratividade de todas estas agências, mostrando que o tamanho não estava necessariamente relacionado com os ganhos financeiros.

Yavas e Fisher (2005) em seu estudo analisaram o desempenho de 31 agências de um grande banco da Califórnia, aplicando DEA na análise da transformação de insumos tais como área disponível e funcionários em produtos tais como receitas e níveis de depósitos e empréstimos. Os resultados mostraram que 22 agências necessitavam de melhorias na relação outputs/inputs.

No trabalho de Golany e Storbeck (1999) encontra-se uma análise de desempenho de 182 agências de um grande banco americano aplicando DEA a informações tais como volume de empréstimos e de depósitos, como outputs, e informações de pessoal e de máquinas de auto-atendimento, como inputs. Os resultados mostram que mais de $50 \%$ das agências atingiram eficiência de 100 $\%$. Além disso, disso os autores acreditam que a DEA é uma das melhores metodologias para análise de eficiência de agências bancárias, pois esta é capaz de considerar múltiplas perspectivas de desempenho, nas quais os gestores acreditam que seu negócio precisa competir.

Zenios et al (1999) conduziram um trabalho onde analisavam a performance de agências bancárias, com o objetivo de eleger benchmarks com base na análise relativa do desempenho das agências, bem como gerar uma guia de melhorias a serem implementadas pela gerência. Os resultados mostraram, com base na análise da relação entre os principais inputs e outputs das agências sob análise, não só as principais fontes de ineficiência, como também as principais recomendações para prover melhorias.

No único trabalho encontrado no Brasil, Macedo et al (2008) aplicaram DEA a pontuação de seis indicadores das perspectivas do BSC de 50 de agências de uma mesma instituição bancária. Os resultados mostraram que apenas 10 agências são tidas realmente como eficientes, servindo assim de padrão de referência (benchmark) para as outras, pois estas possuem nível de desempenho de tal ordem que não necessitam de melhorias. Além disso, o indicador da perspectiva financeira se apresenta com um grande potencial de melhoria para incrementar $o$ desempenho multicriterial das agências sob análise e, por conseguinte, do banco como um todo. 


\section{Metodologia da Pesquisa}

Esta pesquisa pode ser caracterizada, de acordo com Vergara (2009), como sendo descritiva e quantitativa, pois procura-se através da aplicação da Análise Envoltória de Dados (DEA) às informações das agências sob análise, expor características a respeito da performance multicriterial destas.

O processo de amostragem é não probabilístico, pois parte-se de um universo naturalmente restrito, já que as agências foram escolhidas a partir do banco foco deste estudo. Este banco está listado entre os maiores do Brasil, possuindo agências espalhadas em várias partes do território nacional. A formação da amostra se deu por acessibilidade e de forma intencional, porém respeitando as características das agências de forma que a amostra fosse o mais homogênea possível, o que reforçaria a modelagem DEA. Neste sentido, foram coletadas informações de 38 agências pertencentes a uma mesma unidade gerencial, de modo que estas atuam no varejo numa mesma região geográfica, atendendo assim um público alvo bem parecido. Cabe ressaltar que a unidade gerencial escolhida é aquela com menor dispersão geográfica, sendo que suas agências ficam todas num raio de $4 \mathrm{~km}$.

De cada uma das agências foram coletadas informações relativas a perspectivas de desempenho diferentes. A idéia era ter um conjunto de variáveis que conseguisse expressar o desempenho das agências de forma multicriterial, de modo a abranger o maior número possível de dimensões da performance das mesmas. Para tanto, o modelo de perspectivas do BSC foi fundamental, pois inspirados nestas dimensões procurou-se informações disponíveis, que conjugadas pudessem abranger o maior espectro possível do desempenho. Por fim, as dimensões contempladas foram: financeira, operacional, de clientes e de pessoal.

Conforme abordado pelo BSC, para uma boa análise de desempenho é necessário não só que múltiplas perspectivas estejam contempladas, mas sejam consideradas de forma balanceada. Neste sentido, optou-se por colocar cada uma das perspectivas descritas anteriormente com um peso de $25 \%$ na análise do desempenho. Estas perspectivas foram compostas por cinco indicadores, conforme descrito a seguir:

- Quantidade de Funcionários para Atendimento ao Cliente (QTFUN): Representa a quantidade de funcionários que atuam em cada agência. Foi considerado o conjunto de funcionários, que atuam no atendimento ao cliente, pois a satisfação deste depende diretamente deste fator, que interfere em questões como tempo de atendimento.

- Qualidade da Mão-de-Obra para Atendimento aos Clientes (QLFUN): Representa o percentual de funcionários que cumpriram 40 horas de 
treinamento no semestre. Este indicador mede o quanto cada unidade está investindo na formação/qualificação de seus funcionários.

- Nível de Despesas Operacionais (DESPOP): Representa o total de despesas de cada unidade, sem considerar despesas com Provisão para Créditos de Liquidação Duvidosa (PCLD).

- Resultado Financeiro (RESFIN): Representa o Resultado Gerencial de cada unidade. Isto é, o Resultado Operacional descontado a PCLD e Amortizações.

- Nível de Satisfação dos Clientes (SATCL): Representa a nota alcançada por cada agência a partir da não-ocorrência de reclamações ou denuncias e, quando estas ocorrerem, é ponderado também o tempo de atendimento às mesmas. Portanto, trata-se de uma régua de começa com pontuação máxima e vem diminuindo conforme aconteçam ocorrências ou estas venham a ter atendimento demorado.

Estas foram as variáveis utilizadas no modelo de avaliação do desempenho organizacional multicriterial aplicado às agências bancárias sob análise, por se tratarem dos principais elementos de uma análise de eficiência em agências bancárias, conforme pode ser visto nos estudos apresentados no item 3 deste trabalho.

Porém, além destas informações utilizou-se a base de contas de pessoa física (BCPF) como uma proxy de tamanho e nível de atividade das agências. Esta foi utilizada para neutralizar o efeito tamanho. Por exemplo, em nosso modelo quanto maior for o resultado financeiro melhor é o desempenho da agência. Porém, é de se esperar que agências maiores tenham, pelo menos em média, resultados maiores. Por isso, a necessidade de considerar uma medida na qual o efeito tamanho não tivesse impacto. Além disso, no final da análise esta variável foi utilizada para verificar a importância e o impacto do tamanho sobre o desempenho.

As três primeiras variáveis foram tratadas na modelagem como inputs, ou seja, indicadores do tipo quanto menor, melhor o desempenho. Já as duas últimas foram tratadas como outputs (indicadores do tipo quanto maior, melhor o desempenho). Busca-se, portanto, analisar o desempenho tendo como base atingir o máximo de outputs, com a utilização do mínimo de inputs. Cabe ressaltar que as variáveis QTFUN, DESPOP e RESFIN tiveram seus valores originais divididos pelo nível de atividade das agências.

As informações, de cada uma das agências, utilizadas na análise podem ser vistas no quadro 1 , a seguir: 
Quadro 1: Informações das Agências

\begin{tabular}{|c|c|c|c|c|c|c|c|c|c|c|c|}
\hline Agência & QTFUN & DESPOP & QLFUN & RESFIN & SATCL & Agência & QTFUN & DESPOP & QLFUN & RESFIN & SATCL \\
\hline 1 & 4,23 & 137,12 & 46,15 & 432,43 & 47 & 20 & 1,12 & 36,92 & 58,33 & 101,82 & 38 \\
\hline 2 & 1,92 & 50,44 & 50,00 & 116,01 & 37 & 21 & 1,66 & 78,57 & 93,33 & 387,82 & 45 \\
\hline 3 & 3,55 & 17,54 & 57,14 & 35,60 & 47 & 22 & 3,46 & 147,95 & 72,72 & 455,36 & 43 \\
\hline 4 & 2,01 & 90,39 & 64,70 & 287,32 & 43 & 23 & 2,22 & 69,46 & 63,63 & 192,10 & 47 \\
\hline 5 & 3,95 & 161,35 & 57,14 & 496,63 & 29 & 24 & 1,76 & 65,51 & 50,00 & 343,54 & 37 \\
\hline 6 & 3,41 & 173,58 & 80,00 & 351,16 & 47 & 25 & 1,74 & 85,53 & 40,90 & 541,35 & 40 \\
\hline 7 & 1,86 & 48,06 & 56,25 & 127,75 & 42 & 26 & 2,41 & 64,81 & 67,56 & 220,80 & 47 \\
\hline 8 & 2,88 & 81,26 & 60,00 & 207,50 & 43 & 27 & 2,93 & 76,43 & 59,09 & 200,09 & 31 \\
\hline 9 & 2,62 & 57,92 & 68,88 & 187,86 & 36 & 28 & 2,48 & 19,54 & 64,70 & 36,89 & 47 \\
\hline 10 & 2,85 & 89,02 & 76,47 & 243,22 & 46 & 29 & 3,00 & 96,74 & 76,92 & 365,62 & 46 \\
\hline 11 & 5,00 & 100,88 & 62,50 & 174,44 & 47 & 30 & 2,46 & 68,65 & 58,82 & 171,58 & 34 \\
\hline 12 & 2,85 & 58,99 & 92,59 & 124,88 & 47 & 31 & 2,80 & 88,33 & 62,79 & 223,63 & 47 \\
\hline 13 & 2,92 & 77,69 & 72,72 & 199,76 & 41 & 32 & 3,63 & 118,44 & 77,77 & 420,60 & 47 \\
\hline 14 & 2,42 & 67,85 & 77,77 & 160,26 & 47 & 33 & 3,00 & 68,46 & 60,00 & 132,86 & 43 \\
\hline 15 & 2,24 & 63,89 & 58,33 & 190,19 & 47 & 34 & 2,39 & 81,71 & 68,96 & 277,75 & 46 \\
\hline 16 & 3,04 & 9,60 & 87,50 & 30,23 & 47 & 35 & 1,92 & 48,07 & 73,91 & 187,98 & 47 \\
\hline 17 & 2,98 & 126,93 & 71,42 & 362,76 & 47 & 36 & 2,06 & 45,51 & 33,33 & 106,19 & 44 \\
\hline 18 & 2,88 & 127,28 & 83,33 & 349,86 & 47 & 37 & 3,15 & 128,78 & 83,33 & 366,80 & 47 \\
\hline 19 & 1,92 & 63,79 & 62,50 & 247,05 & 47 & 38 & 2,61 & 110,95 & 80,00 & 312,88 & 47 \\
\hline
\end{tabular}

Fonte: Dados da pesquisa.

Em uma análise de desempenho, ressaltam Gomes et al. (2002), pode-se utilizar uma análise monocritério ou uma multicritério. Na Análise Monocritério, que neste caso pode ser expressa por cada um dos índices listados, pode-se valorar cada ação potencial sobre um único eixo de significância, ou seja, uma unidade comum. Já na Análise Multicritério tem-se um novo paradigma que reconhece a heterogeneidade das dimensões de desempenho, através da utilização de uma família de múltiplos critérios, escolhidos segundo as exigências técnicas, inteligibilidade, aceitação como base do trabalho e facilidade de utilização como instrumento de análise.

Neste artigo, para consolidar o desempenho sob as múltiplas perspectivas consideradas, utiliza-se uma ferramenta de Análise Multicritério: a Análise Envoltória de Dados (DEA). Com esta é possível avaliar o desempenho de cada agência de modo multicriterial, ou seja, considerando de maneira integrada todos os vetores de desempenho apresentados (cada índice individualmente), obtendo o que denominou-se de Índice de Desempenho Multicriterial (IDM).

Segundo Cooper et al (2004) a metodologia DEA tem sua origem com o trabalho de Farrell (1957), que propôs uma abordagem de análise que pudesse ser mais adequada à gestão de qualquer organização produtiva. Já os modelos DEA, propriamente ditos, tiveram seu início em 1978 com a tese de Ph.D de Edwardo Rhodes sob a orientação de W.W. Cooper. Em seu trabalho, Rhodes ampliou o trabalho de Farrell analisando unidades que possuíam mais de um input e/ou 
output.

A metodologia DEA foi inicialmente desenvolvida no modelo de Retornos Constantes de Escala (CRS - Constant Returns to Scale), também conhecido por CCR (Charnes, Cooper e Rhodes). Este modelo determina uma fronteira CRS que indica que crescimento proporcional dos inputs produzirá crescimento proporcional dos outputs. Este modelo tem como propriedades a convexidade, o cálculo da ineficiência e o raio ilimitado (que presume a proporcionalidade entre inputs e outputs).

Depois, em 1984, foi desenvolvido o modelo BCC (Banker, Charnes e Cooper) ou VRS (Variable Returns to Scale), que assume rendimentos crescentes e decrescentes de escala na fronteira de eficiência. Este modelo surgiu como resultante da partição da eficiência do modelo CCR em duas componentes: a eficiência técnica (VRS) e a eficiência de escala (CRS/VRS).

Soares de Mello et al (2005) definem DEA como uma metodologia matemática não-paramétrica baseada em programação linear para definição de eficiência de DMU's (Decision Making Units) homogêneas. Para tanto, a DEA utiliza um único indicador de desempenho para cada unidade analisada, a partir da relação ponderada entre inputs e outputs. De maneira geral, continuam os autores, a metodologia DEA identifica as DMU's eficientes, mede e localiza a ineficiência; fornece o benchmark para as unidades ineficientes; e identifica a origem da ineficiência relativa de cada DMU.

De acordo com Charnes et al. (1994) e Coelli et al. (1998), a Análise Envoltória de Dados (DEA), mostra o quão uma empresa é eficiente, no tratamento de seus inputs e outputs, em relação às outras, numa análise que fornece um indicador que varia de 0 a 1 ou de $0 \%$ a $100 \%$. Somente as empresas que obtêm índice de eficiência igual a um é que fazem parte da fronteira eficiente.

Lins e Meza (2000) ressaltam que um caminho intuitivo para introduzir DEA é por meio de forma de razão. Para cada DMU, procura-se obter uma medida de razão de todos os outputs sobre todos os inputs. Ou seja, a modelagem procura encontrar os pesos ótimos uj e vi para a resolução do seguinte problema de programação matemática.

$$
\begin{aligned}
\operatorname{Max} E_{c}= & \frac{\sum_{j=1}^{s} u_{j} y_{\dot{x}}}{\sum_{i=1}^{m} v_{i} x_{i}} \\
\text { S.a.: } \quad & \frac{\sum_{j=1}^{s} u_{j} y_{k}}{\sum_{i=1}^{m} v_{i} x_{k}} \leq 1, k=1,2, \ldots, c, \ldots, n \\
& u_{j} \geq 0, \forall j, \\
& v_{i} \geq 0, \forall i
\end{aligned}
$$


Neste estudo foi utilizado o modelo DEA-CRS, porém com uma condição especial de restrição aos pesos. A seguir tem-se a modelagem utilizada na análise do desempenho multicriterial, já considerando a proposta de Charnes e Cooper (1962), para linearizar a relação apresentada anteriormente, que era um problema de programação fracionária:

$$
\begin{aligned}
\operatorname{Max} E_{c}= & \sum_{j=1}^{s} u_{j} y_{\dot{i}} \\
\text { S.a.: } \quad & \sum_{i=1}^{m} v_{i} x_{i}=1 \\
& \sum_{j=1}^{s} u_{j} y_{k}-\sum_{i=1}^{m} v_{i} x_{k} \leq 0, k=1,2, \ldots, c, \ldots, n \\
& u_{j}, v_{i} \geq 0, \forall x, y .
\end{aligned}
$$

Neste modelo, c é a unidade (DMU - Decision Making Units) que está sendo avaliada. O problema acima envolve a procura de valores para $u_{j}$ e $v_{i}$, que são os pesos, de modo que maximize a soma ponderada dos outputs $\left(\mathrm{y}_{\mathrm{j}}\right.$ ) da DMU em estudo, sujeita as restrições de que a soma ponderada dos inputs $\left(\mathrm{x}_{\mathrm{i})}\right.$, desta mesma DMU, seja igual a um e a diferença entre a soma ponderada dos outputs $\left(\mathrm{y}_{\mathrm{j}}\right.$ e a soma ponderada dos inputs $\left(\mathrm{x}_{\mathrm{i}}\right.$ ) seja menor ou igual a zero, para todas as DMU's. Esta última restrição faz com que quando o mesmo conjunto de coeficientes de entrada e saída (os vários $u_{j}$ e $v_{i}$ for aplicado a todas as outras unidades que estão sendo comparadas, nenhuma unidade excederá $100 \%$ de eficiência.

Esta forma de PPL (Problema de Programação Linear) é conhecida como problema dos multiplicadores, como também são chamados os pesos, $u_{j}$ e $v_{i}$ e será utilizada nesta análise para calcular o desempenho multicriterial de cada agência. Macedo et al. (2006) dizem que neste modelo, para cada DMU a ser analisada, formula-se um problema de otimização com o objetivo de determinar os valores que esta DMU atribuiria aos multiplicadores $u_{j}$ e $v_{i}$ (importância relativa de cada variável/critério), de modo a aparecer com a maior eficiência possível. Neste sentido, para se obter importâncias balanceadas, utilizou-se de um artifício matemático de limitar ou restringir a atribuição dos pesos pelo modelo, de modo que cada perspectiva ficasse no final com o mesmo peso/participação no Índice de Desempenho Multicriterial (IDM).

Relaciona-se, a seguir, algumas características da DEA, destacadas por Lins e Meza (2000):

- $\quad$ Não necessita da conversão das variáveis de inputs e outputs em valores monetários, o que a diferencia dos métodos baseados em avaliação puramente econômica;

- Os índices de eficiência são baseados em dados reais (e não em 
fórmulas teóricas);

- É uma alternativa e um complemento a análise de tendência central e análise de custos;

- Considera a possibilidade de não se descartar os outliers como desvios em relação ao comportamento "médio", pois estes podem ser modelos de eficiência a serem estudados pelas demais DMU's.

- Otimiza cada observação individual a fim de determinar uma fronteira por partes que compreende o conjunto de DMU's Pareto-eficientes, o que não ocorre nos modelos paramétricos tradicionais.

A utilização da DEA pressupõe alguns cuidados essenciais. A capacidade de definir DMU's eficientes e ineficientes é muito sensível ao número de inputs e outputs. Ao aumentar o número de variáveis há uma tendência de se aumentar a média de eficiência do modelo. Soares de Mello et al (2005) recomendam que o número de DMU's seja pelo menos 3 vezes maior que a soma dos números de inputs e outputs. Os autores ressaltam, ainda que a análise conduzida pela DEA restringe-se a amostra analisada, o que não permite a comparação com estudos distintos. Isto é, os resultados obtidos são validos somente para as variáveis e as DMU's do estudo.

Por fim, cabe destacar que para obter-se o indicador de desempenho consolidado com base na DEA, utilizou-se um software específico denominado Frontier Analist 4.0 da Banxia Software. Esta é uma ferramenta capaz de calcular a eficiência das organizações com base na metodologia DEA (BCC e/ou CCR), com possibilidade de restrição aos pesos.

Depois de obtido os índices de desempenho multicriteriais, resultado da consolidação feita através da DEA, das várias variáveis das diferentes perspectivas utilizadas, procedeu-se análises complementares do desempenho das agências. Estas análises envolveram o cálculo de correlações e da aplicação de teste não paramétrico de diferença de médias, com o intuito de verificar a relação entre as variáveis e o desempenho.

O teste aplicado foi o de Mann-Whitney, ao nível de $5 \%$ de significância, com o objetivo de verificar a existência de diferença estatisticamente significativa entre o desempenho das 10 maiores e das 10 menores agências (considerando a base de contas de pessoa física como proxy de tamanho). Segundo Siegel e Castellan Jr. (2006), este teste é uma boa alternativa ao teste paramétrico t, quando se deseja evitar as suposições do mesmo, como a necessidade de distribuição normal (já que tem-se poucos dados).

Além disso, para analisar a relação entre as variáveis e as mesmas com o desempenho multicriterial, fez-se uma análise de correlação, tanto de Pearson, quanto de Spearman. De acordo com Siegel e Castellan Jr. (2006), a correlação 
de Spearman é uma medida de associação entre duas variáveis que requer que ambas as variáveis sejam medidas pelo menos em uma escala ordinal, de modo que os objetos ou indivíduos em estudo possam ser dispostos em postos em duas séries ordenadas. Já a correlação de Pearson, segundo Stevenson (1981), sintetiza o grau de relacionamento entre duas variáveis contínuas. Logo, foi analisada a correlação de Pearson entre os valores das variáveis e entre estes e o valor do IDM. Já análise da correlação de Spearman foi feita entre as ordenações das variáveis e a ordenação do IDM.

\section{Análise dos Resultados}

De posse dos dados disponíveis de cada agência utilizou-se o aplicativo Frontier 4.0 da Banxia Software, para rodar a DEA, que gerou as informações disponíveis no quadro 02 sobre o desempenho multicriterial (Índice de Desempenho Multicriterial - IDM) das agências sob análise. Cabe lembrar, que este foi calculado considerando o mesmo peso para todas as perspectivas, logo as variáveis QTFUN e QLFUN ficaram com peso de 12,5\%, já que eram ambas da perspectiva de pessoal, enquanto cada uma das outras três variáveis ficou com peso de $25 \%$.

\section{Quadro 02: Desempenho das Agências}

\begin{tabular}{|c|c|c|c|c|c|}
\hline Agência & IDM & Agência & IDM & Agência & IDM \\
\hline 1 & 60,53 & 14 & 44,54 & 27 & 44,11 \\
\hline 2 & 44,49 & 15 & 56,23 & 28 & 32,50 \\
\hline 3 & 34,12 & 16 & 50,28 & 29 & 61,28 \\
\hline 4 & 60,25 & 17 & 53,44 & 30 & 44,97 \\
\hline 5 & 44,89 & 18 & 50,87 & 31 & 49,05 \\
\hline 6 & 41,95 & 19 & 69,73 & 32 & 57,29 \\
\hline 7 & 50,34 & 20 & 54,04 & 33 & 37,57 \\
\hline 8 & 47,91 & 21 & 80,20 & 34 & 60,59 \\
\hline 9 & 52,75 & 22 & 52,42 & 35 & 67,24 \\
\hline 10 & 49,56 & 23 & 53,02 & 36 & 48,54 \\
\hline 11 & 34,12 & 24 & 83,20 & 37 & 50,95 \\
\hline 12 & 38,10 & 25 & 100,00 & 38 & 52,70 \\
\hline 13 & 45,59 & 26 & 60,06 & & \\
\cline { 1 - 4 } & & & & &
\end{tabular}

Fonte: Dados da pesquisa.

Uma análise do Quadro 02 mostra que somente a agência 25 é que atingiu o desempenho máximo. Esta agência, então, passa a ser referências para as outras num processo de benchmarking, pois é uma agência de destaque em termos de desempenho multicriterial, levando-se em conta as perspectivas e variáveis utilizadas na análise. Na verdade, esta agência foi a que melhor combinou os inputs (menores) e outputs (maiores), levando-se em consideração os pesos atribuídos 
aos mesmos.

Cabe destacar que isso é resultado direto do desempenho desta agência em relação às perspectivas consideradas. Ela é a melhor na perspectiva financeira e a segunda melhor na de pessoal.

Observa-se, ainda, que as outras agências possuem desempenho bem inferior à agência 25, pois as melhores depois desta são as agências 24 e 21, com respectivamente, 83,20 e 80,20 de IDM. Estas três agências podem ser consideradas com bom desempenho (IDM $>80$ )

Como destaques negativos (IDM $<40 \%$ ) tem-se as agências 28 (IDM = 32,50), 11 e $3($ IDM = 34,12), 33 (IDM = 37,57) e 12 (IDM = 38,10). Estas são as piores agências da amostra, considerando as perspectivas e as variáveis utilizadas na análise. Elas não conseguem ter uma boa relação output/input, ou seja, ou usam insumos demais e/ou geram produtos de menos.

Como dito anteriormente, com base nestes indicadores de desempenho multicriterial procedeu-se análises de correlação e um teste não paramétrico de diferença de médias. Nas análises de correlação entre as variáveis obteve-se os seguintes resultados significativos:

- Entre BCPF e DESPOP (- 0,471): resultado significativo ao nível de 1 $\%$ que representa ganho de escala na operação das maiores agências, pois quanto maior a agência menor a despesa por conta.

- Entre BCPF e QTFUN (- 0,589): resultado significativo ao nível de 1 $\%$ que também representa ganho de escala na operação das maiores agências, pois quanto maior a agência menor a quantidade de funcionários por conta.

- Entre DESPOP e QTFUN (+ 0,520): resultado significativo ao nível de $1 \%$ que representa uma relação positiva entre o a despesa operacional e o número de funcionários, ou seja, quanto maior o número de funcionários maior a despesa operacional.

- Entre DESPOP e RESFIN (+ 0,818): resultado significativo ao nível de $1 \%$ que mostra que quanto melhor a infra-estrutura gerada pelos gastos com despesas operacionais, maior o desempenho financeiro da agência.

- Entre QLFUN e SATCL (+ 0,416): resultado significativo ao nível de $1 \%$ que mostra que a satisfação dos clientes está diretamente relacionada com a qualidade da mão-de-obra, ou seja, é a qualidade e não a quantidade de funcionários que gera satisfação do cliente.

Em relação ao desempenho multicriterial encontrou-se as seguintes correlações significativas:

- Com a QTFUN (Pearson $=-0,491$ e Spearman $=+0,461)$ : resultados significativos ao nível de $1 \%$ que mostram que quanto maior o número de fun- 
cionários, menor o desempenho.

- Com o RESFIN (Pearson $=+0,569$ e Spearman $=+0,537)$ : resultados significativos ao nível de $1 \%$ que mostram que quanto maior o resultado financeiro, maior o desempenho.

Estes resultados já eram esperados, já que na análise a QTFUN é tratada como um input (quanto menor, melhor o desempenho) e o RESFIN como output (quanto maior, melhor o desempenho). Importante destacar que as outras variáveis não foram significativas nem ao nível de $10 \%$, o que destaca a QTFUN e o RESFIN, como os grandes responsáveis pelo desempenho, apesar da preocupação inicial de balanceamento dos pesos.

Uma análise complementar foi feita para concluir a respeito do efeito tamanho sobre o desempenho. A idéia é verificar a correlação entre esta variável e o IDM e testar a diferença média entre o IDM das maiores e das menores agências. Como dito anteriormente a BCPF é a proxy de tamanho e nível de atividade das agências utilizada.

Nas correlações de Pearson $(0,310)$ e de Spearman $(0,301)$ percebe-se que o desempenho não tem relação significativa com o tamanho ao nível de $5 \%$, mas tem ao nível de $10 \%$. Percebe-se, ainda, pelos valores dos índices que está relação, mesmo que não muito forte, é positiva, indicando alguma relação entre tamanho e desempenho. Isso é reflexo do que foi encontrado em relação ao ganho de escala nas operações das agências.

Isso é ratificado pelo teste não paramétrico de diferença de médias de Mann-Whitney, que mostra que a hipótese nula de que as médias são iguais é aceita ao nível de $5 \%(\operatorname{sig}=0,075)$, ou seja, não existe diferença estatisticamente significativa ao nível de $5 \%$ para o desempenho multicriterial dos dois grupos sob análise (10 maiores e 10 menores agências). Porém, ao nível de $10 \%$ de significância o teste mostra que a hipótese nula de que as médias são iguais é rejeitada, ou seja, o desempenho multicriterial médio das 10 maiores agências (IDMmédio $=61,24)$ é superior ao desempenho multicriterial médio das 10 menores agências $($ IDMmédio $=48,89)$.

\section{Considerações finais}

Com base nos resultados encontrados pode-se concluir que a metodologia DEA foi capaz de analisar de forma multicriterial a performance das agências, consolidando as variáveis das diversas perspectivas de desempenho consideradas, em um único indicador.

Os resultados desse estudo propõem uma nova percepção sobre a performance de unidades de negócios a partir da análise de indicadores que representem 
as perspectivas do BSC, que não se encontram disponíveis aos gestores em geral através dos tradicionais métodos de análise. Ou seja, a partir de informações que não estariam disponíveis pelas técnicas convencionais, os resultados da Análise Envoltória de Dados (DEA) podem proporcionar melhores condições de competitividade ao banco (foco da análise), pela melhoria da atuação de suas agências, principalmente quando interpretadas e usadas com os conhecimentos e julgamentos próprios da alta administração sobre suas operações.

Numa análise dos resultados apresentados, pode-se perceber que a melhor agência (25) é aquela que possui um desempenho multicriterial superior. Isto quer dizer que esta, que possui desempenho relativo superior, pode ser utilizada como benchmark para as demais. Percebe-se, ainda, que as demais agências possuem IDM bem abaixo desta, mostrando a necessidade de mudanças substanciais no gerenciamento das mesmas, tendo como modelo de referência a agência 25 .

$\mathrm{Na}$ análise de correlação entre as variáveis percebe-se que existe um ganho de escala na operação das maiores agências, pois quanto maior a agência menor a despesa por conta e menor a quantidade de funcionários por conta. Observa-se, ainda, que a satisfação dos clientes está diretamente relacionada com a qualidade da mão-de-obra (nível de treinamento), ou seja, é a qualidade e não a quantidade de funcionários que gera satisfação do cliente.

Por fim, nota-se que o desempenho tem relação positiva significativa, mesmo que não muito forte, com o tamanho ao nível de $10 \%$, o que indica alguma relação entre tamanho e desempenho. A comparação entre o desempenho médio das 10 maiores e das 10 menores agências corrobora esta sinalização, já que, ao nível de $10 \%$ de significância, o teste aplicado mostra que o desempenho multicriterial médio das 10 maiores agências é superior ao das 10 menores. Isso é reflexo do que foi encontrado em relação ao ganho de escala nas operações das agências.

Cabe salientar, que este assunto não se encontra encerrado, pois ainda há muito a ser explorado desta metodologia em gestão de instituições financeiras. Além disso, este trabalho representa apenas o início desta trajetória de pesquisa, que terá continuidade através de uma discussão com os gestores das agências sobre a seleção de variáveis para a análise do desempenho, que poderá resultar na aplicação conjunta de outras ferramentas tais como a análise fatorial (quantitativa) ou a técnica Delphi (qualitativa).

Além disso, os resultados do uso da DEA sugerem que outros estudos nesta área de análise de eficiências de unidades de negócios (agências, filiais, concessionárias, etc.) possam ser conduzidos no Brasil.

Agradecimentos: Os autores agradecem o apoio do CNPq a esta pesquisa. 


\section{Referências}

ALMEIDA, S. de; MARÇAL, R. F. M.; KOVALESKI, J. L. Metodologias para Avaliação de Desempenho Organizacional. In: ENCONTRO NACIONAL DE ENGENHARIA DE PRODUÇÃO, 24, 2004, Florianópolis. Anais do XXIV ENEGEP. Florianópolis: ABEPRO, 2004. 1 CD.

ATKINSON, A. A.; BANKER, R. D.; KAPLAN, R. S.; YOUNG, S. M. Contabilidade Gerencial. São Paulo: Atlas, 2000.

AVKIRAN, N. K. An application reference for data envelopment analysis in branch banking: helping the novice researcher. International Journal of Bank Marketing. v. 17, n. 5, p. 206-220, 1999.

BANKER, R. D.; CHARNES, A.; COOPER, W. W. Some Models for Estimating Technical and Scale Inefficiencies in Data Envelopment Analysis. Management Science. v. 30, n. 9, 1078-1092. 1984.

CAMANHO, A. S.; DYSON, R. G. Cost efficiency, production and value-added models in the analysis of bank branch performance. Journal of the Operational Research Society. v. 56, n. 5, p. 483-494, 2005.

CHARNES, A.; COOPER, W. W.; RHODES, E. Measuring the Efficiency of Decision Making Units. European Journal of Operational Research. v. 2, n. 6, 429-444. 1978.

CHARNES, A.; COOPER, W. W. Programming with Linear Fractional Functionals. Naval Research Logistic Quarterly, v. 9, p. 181-186, 1962.

CHARNES, A.; COOPER, W. W.; LEWIN, A. Y.; SEIFORD, L. M. Data Envelopment Analysis. 2. ed. Boston: KAP, 1994.

CHEN, T.; CHEN, L.DEA performance evaluation based on BSC indicators incorporated: The case of semiconductor industry. International Journal of Productivity and Performance Management. v. 56, n. 4, p. 335-357, 2007.

COELLI, T.; RAO, D. S. P.; BALTESE, G. E. An Introduction to Efficiency and Productivity Analysis. Boston: KAP, 1998.

COOPER, W. W.; SEIFORD, L. M.; ZHU, J. Handbook on Data Envelopment Analysis. Boston: KAP, 2004.

EILAT, H.; GOLANY, B.; SHTUB, A. R\&D project evaluation: An integrated 
DEA and Balanced Scorecard approach. Omega, v. 36, n. 5, p. 895-912, 2006.

ESPOSTO; K. F.; GEROLAMO, M. C.; RENTES, A. F. Sistema de Medição de Desempenho - Uma Proposta Conceitual. In: ENCONTRO NACIONAL DE ENGENHARIA DE PRODUÇÃO, 22, 2002, Curitiba. Anais do XXII ENEGEP. Curitiba: ABEPRO, 2002. 1 CD.

FERRAZ, C. A.; MARTINS, R. A. Um Método Abrangente para o Diagnóstico da Medição de Desempenho. In: ENCONTRO NACIONAL DE ENGENHARIA DE PRODUÇÃO, 22, 2002, Curitiba. Anais do XXII ENEGEP. Curitiba: ABEPRO, 2002. 1 CD.

GOLANY, B.; STORBECK, J. E. A Data Envelopment Analysis of the Operational Efficiency of Bank Branches. Interfaces. v. 29, n. 3, p. 14-26, 1999.

GOMES, L. F. A. M.; GOMES, C. F. S.; ALMEIDA, A. T. Tomada de Decisão Gerencial: enfoque multicritério. São Paulo: Atlas, 2002.

GOMES, L. F. A. M.; GONZALEZ-ARAYA, M. C.; CARIGNANO, C. Tomada de decisões em cenários complexos. Rio de Janeiro: Pioneira Thompson Learning. 2004.

KAPLAN, R. S.; NORTON, D. P. A Estratégia em Ação: Balanced Scorecard. Rio de Janeiro: Campus-Elsevier, 1997.

LINS, M. P. E.; MEZA, L. A. Análise Envoltória de Dados e perspectiva de integração no ambiente de apoio à decisão. Rio de Janeiro: COPPE/UFRJ, 2000 .

MACEDO, M. A. S.; FARIAS DA SILVA, F.; MELO SANTOS, R. Análise do Mercado de Seguros no Brasil: uma visão do desempenho organizacional das seguradoras no ano de 2003. Revista Contabilidade \& Finanças. Edição Especial - Atuária, 2006.

MACEDO, M. A. S.; CASA NOVA, S. P. C.; ALMEIDA, K. Mapeamento e Análise Bibliométrica da Utilização da Análise Envoltória de Dados (DEA) em Estudos das Áreas de Contabilidade e Administração. In: ENCONTRO DA ASSOCIAÇÃO NACIONAL DE PÓS-GRADUAÇÃO E PESQUISA EM ADMINISTRAÇÃO, 31, 2007, Rio de Janeiro. Anais do XXXI ENANPAD. Rio de Janeiro: ANPAD, 2007. 1 CD.

MACEDO, M. A. S.; BARBOSA, A. C. T. A. M.; CAVALCANTE, G. T. Desempenho de Agências Bancárias no Brasil: aplicando Análise Envoltória de Dados 
(DEA) a indicadores relacionados às perspectivas do BSC. In: CONGRESSO USP DE CONTROLADORIA E CONTABILIDADE, 8, 2008, São Paulo. Anais do VIII Congresso USP de Controladoria e Contabilidade. São Paulo: EACFEA-USP, 2008. 1 CD.

MARR, B.; SCHIUMA, G. Business Performance Measurement - past, present and future. Management Decision, v. 41, n. 8, p. 608-687, 2003.

MELLO, João Carlos C. B. S. de et al. Curso de Análise Envoltória de Dados. In: SIMPÓSIO BRASILEIRO DE PESQUISA OPERACIONAL, 37, 2005, Gramado. Anais do XXXVII SBPO. Gramado/RS: SOBRAPO, 2005. 1 CD.

NEELY, A.; GREGORY, M.; PLATTS, K. Performance measurement system design - A literature review and research agenda. Internacional Journal of Operations \& Production Management, v. 25, n. 12, p. 1226-1263, 2005.

RICKARDS, R. Setting benchmarks and evaluating Balanced Scorecards with Data Envelopment Analysis. Benchmarking: An International Journal. v. 10, n.3, p. 226-245, 2003.

ROCHA, A. M. C.; CARNEIRO, J.; SILVA, J. F.; HEMAIS, C. A. Mensuração do desempenho organizacional: questões conceituais e metodológicas. In: GUTIERREZ, M.; BERTRAND, H. (Orgs.). Estudos em Negócios IV. 1 ed. Rio de Janeiro: Mauad, 2005, v. 1, p. 58-79.

SANTOS, A.; CASA NOVA, S. P. C. Proposta de um Modelo Estruturado de Análise de Demonstrações Contábeis. RAE-eletrônica. v. 4, n. 1, art. 8, 2005. Disponível em: <http://www.rae.com.br/eletronica>. Acesso em: 13 jan. 2008.

SIEGEL, S.; CASTELLAN Jr., N. J. Estatística Não-Paramétrica para Ciências do Comportamento. 2 ed. Porto Alegre: Artmed, 2006.

SOTERIOU, A. C.; STAVRINIDES, Y. An internal customer service quality data envelopment analysis model for bank branches. International Journal of Operations \& Production Management. v. 17, n. 8, p. 780-789, 1997.

STEVENSON, W. J. Estatística Aplicada à Administração. São Paulo: Harbra, 1981.

VERGARA, S. C. Projetos e Relatórios de Pesquisa em Administração. 10. ed. São Paulo: Atlas, 2009.

WANG, J. Corporate Performance Efficiency Investigated by Data Envelopment Analysis and Balanced Scorecard. Journal of American Academy of Business. 
v. 9, n. 2, p.312-318, 2006.

YAVAS, B. F.; FISHER, D. M. Performance Evaluation of Commercial Bank Branches using Data Envelopment Analysis. Journal of Business and Management. v. 11, n. 2, p. 89-102, 2005.

ZENIOS, C. V.; ZENIOS, S. A.; AGATHOCLEOUS. K.; SOTERIOU, A. C. Benchmarks of the Efficiency Bank Branches. Interfaces. v. 29, n. 3, p. 37-51, 1999. 
